\title{
Case Report: Hemoptysis as a Presentation of Aortic Dissection
}

\author{
Dejan Marčetić ${ }^{1}$, Ana Nedić*1 and Alenka Borovčak Lovrenc ${ }^{2}$ \\ ${ }^{1}$ Department of Internal Medicine, Croatia \\ ${ }^{2}$ Department of Radiology, Croatia
}

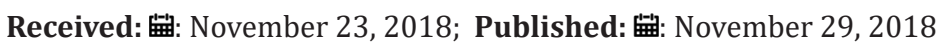

*Corresponding author: Ana Nedić , Department of Internal Medicine, Gajeva 21, Virovitica 33000, Croatia

\section{Introduction}

Hemoptysis is the expectoration of blood originating from the lower respiratory tract, from a source below the glottis. It is a common alarming symptom for patients and pulmonologists. The most common causes of hemoptysis are bronchiectasis, pulmonary tuberculosis, necrotizing pneumonia, abscesses, bronchogenic carcinoma, bronchitis, carcinoid or hemoptysis of unknown etiology. A rare causes of hemoptysis are pulmonaryrenal syndrome, congenital anomalies, pulmonary embolism and pulmonary infarction [1-4]. Other causes are coagulation disorders, heart failure, aortic aneurysm and certain medications. Aortic dissection describes the condition when a separation has occurred in aortic wall intima, causing blood flow into a new false channel composed of the inner and outer layers of the media. Dissection most commonly occurs with a discrete intimal tear, but can occur without one [6]. The most common symptoms are shortness of breath, loss of consciousness, sudden severe upper back or chest pain often described as a "tearing" sensation from the neck down the back. The early diagnosis of aortic dissection improves treatment and outcome [7].

\section{Case Report}

A 81-year-old female was admitted to the emergency department (ED) of general hospital due to expectoration of nonsignificant amount of fresh blood a day prior to the admission. She also complained about increasing cough intensity and excessive sweating. In addition she reported the diffuse chest pain and interscapular back pain duration of 14 days. The patient associated the pain with a trauma two weeks ago when she fell of the bed facing down and hit the chest. Past medical history included arterial hypertension, coronary heart disease with one stent implantation a year ago, rheumatoid arthritis, dyslipidemia, epilepsy and implantation of total endoprosthesis of one knee and hip. Otherwise, she is treated abroad so she was not able to submit any earlier medical documentation. Current medications included candesartan (8mg twice daily), pantoprazole (20mg once daily), methotrexate (7.5mg per week), prednisolone $(2 \mathrm{mg}$ and $1 \mathrm{mg}$ alternately), adalimumab (40mg every two weeks subcutaneously), lamotriginum ( $25 \mathrm{mg}$ twice daily) furosemide with potassium citrate (40mg and one tablet every other day, respectively), acetylsalicylic acid (100mg once daily), and for the last week she used antibiotic and antitussive drug with a poor response. On presentation she was conscious, well-oriented in all directions, afebrile, with restricted mobility due to the trauma 14 days ago. She was tachycardic with heart rate 110 beats/minute, and had systolic murmur all over precordium, intensity III-IV.

Auscultation of the lungs revealed rare bilateral basal crackles. The blood pressure was $180 / 90 \mathrm{mmHg}$, respiratory rate was 13 breaths/minute and oxygen saturation was $93 \%$ on room air. Abdominal exam was unremarkable while there was a mild perimaleroral edema on both legs. Initial laboratory evaluation showed mild normocytic anemia (hemoglobin 105g/L), elevated white blood cells (14.7 x 109/L), neutrophils (12 x 109/L), C-reactive protein $(283.7 \mathrm{mg} / \mathrm{L})$, and mildly elevated cardiac troponin $\mathrm{T}$ (22.63ng/L). Other laboratory test were unremarkable. ECG showed sinus rhythm with heart frequency 110 beats/minute and incomplete right bundle branch block. Other ECG parameters were within limits. The chest radiograph demonstrated heartenlargement with hypertrophic configuration and aortic calcifications while on the lungs there was a mild left laterobasal pleural effusion. She was admitted to the Department of Pulmonology where she was treated with fluoroquinolone antibiotics and symptomatic therapy. Next morning the patient complained about very intensive interscapular back pain with progression to the left shoulder. During the night she had two attacks of hemoptysis (about $10 \mathrm{ml}$ ). She also reported meteorism and constipation for the last five days which is not usual for her. 
On examination she was tachypnoic, excessive sweated, afebrile, paled. Blood pressure was high $(180 / 90 \mathrm{mmHg})$, respiratory rate was 25 breaths per minute, oxygen saturation was $93 \%$ on room air. Auscultation of lungs revealed reduced breath sounds at the left basal parts. ECG showed sinus tachycardia with heart rate 112 beats per minute and incomplete right bundle branch. Multiline CT pulmonary angiography was performed and excluded pulmonary embolism but revealed thoracic aortic aneurysm beneath the origin of the left subclavian artery. Dimensions in the middle part of thoracic aorta were $44 \times 51 \mathrm{~mm}$. According to classification criteria it was Standford B, De Bakey III. The thick thrombus, $15 \mathrm{~mm}$ width, was placed around entire D aneurysmal circumference and had a few ulcerations with the biggest one $65 \mathrm{~mm}$ beneath the orifice of the left subclavian artery, placed dorsal and parasagittal with neck width $19 \times 11 \mathrm{~mm}$ and total diameter $24 \times 17 \mathrm{~mm}$. Also, it was seen an in homogenous structure with hyperdense areas inside the thrombus in an aneurysm which primarily shows dissecting aneurysm with intermittent and probably recent bleeding in the thrombus. The aneurysm ends intrathoracic on the level of the lower left pulmonary vein and according to the outer line of the aortic arch the length of the aneurysm is $11,7 \mathrm{~cm}$. Also there were compressive atelectasis Para mediastinal in the parenchyma of the left lung, probably as a consequence of the compressive effect of the aneurysm. The patient was immediately admitted to the Intensive Care Unit due to monitoring vital parameters. ACE inhibitors and beta blockers were administered because of the prevention of attacks of high blood pressure. Opioid analgesic therapy was unsuccessful, moreover the patient reported "tearing" pain with progression to the left shoulder and the abdomen with the distension of the abdomen. Also she became febrile ( $38{ }^{\circ} \mathrm{C}$ ) with hemoptysis still present. According to her physical status and CT analysis she was urgently transferred to the Special hospital for cardiovascular surgery where an endovascular stent graft was successfully implanted using Thoracic Endovascular Aortic Repair (TEVAR) procedure.

\section{References}

1. Naidich DP, Funtk S, Ettenger N, Arranda C (1990) Haemoptysis: CT bronchoscopic correlation in 58 cases. Radiology 177(2): 357-362.

2. Santiago S, Tobias J, Williams AJ (1991) A reappraisal of the causes of haemoptysis. Arch Intern Med 151(12): 2449-2451.

3. Hirshberg B, Biran I, Glazer M, Merdechai R (1997) Haemoptysis: aetiology, evaluation and autcome in a tertiary referal Hospital. Chest 112(2): 440-444.

4. Abal AT, Nair PC, Cherian J (2001) Haemoptysis: aetiology, evaluation and autcome - prospective study in a third-world country. Respiratory medicine 95(7): 548-552.

5. Stoller JK (1992) Diagnosis and management of massive haemoptysis: a review. Respir care 37(6): 564-581.

6. Lerson EW, Edwards WD (1984) Risk factors for aortic dissection: A necropsy study of 161 cases. Am J Cardiol 53(6): 849-855.

7. Cigarroa JE, Isselbacher EM, DeSantic RW, Eagle KA (1993) Diagnostic imaging in the evaluation of suspected aortic dissection. Old standards and new directions. N Engl J Med 328(1): 35-43.

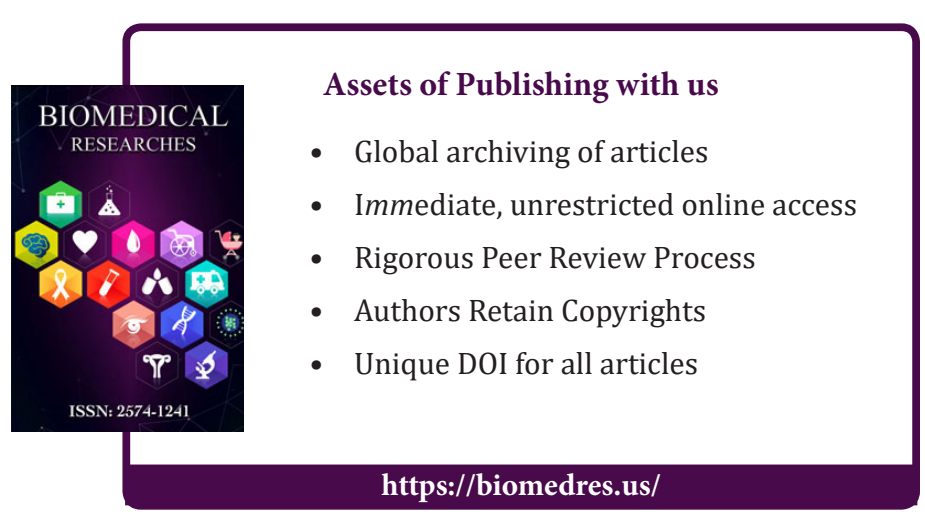

\section{ISSN: 2574-1241}

DOI: $10.26717 / B J S T R .2018 .11 .002122$

Ana Nedić Biomed J Sci \& Tech Res

(C) Commons Attribution 4.0 License

Submission Link: https://biomedres.us/submit-manuscript.php 